\title{
Equality of opportunity, moral hazard and the timing of luck
}

\author{
Arnaud LEFRANC* \\ Alain TRANNOY
}

Wednesday $12^{\text {th }}$ April, 2017

\begin{abstract}
Equality of opportunity is usually defined as a situation where the effect of circumstances on outcome is nullified (compensation principle) and effort is rewarded (reward principle). We propose a new version of the reward principle based on the idea that effort deserves reward for it is costly. We show that luck can be introduced in two ways in the definition of these principles, depending on whether the correlation between luck and circumstances should be nullified and whether the correlation between luck and effort should be rewarded. In this regard, the timing of luck with respect to effort decisions is crucial, as is exemplified by moral hazard where effort choice influences the lottery of future uncertain events.
\end{abstract}

JEL codes: D63,J62,C14

Keywords: equality of opportunity, effort, luck, timeline, moral hazard, reward.

${ }^{*}$ University of Cergy-Pontoise, THEMA and IZA

${ }^{\dagger}$ Aix-Marseille University(Aix-Marseille School of Economics, CNRS and EHESS.

${ }^{\ddagger}$ We are grateful to Marc Fleurbaey and two anonymous referees for useful suggestions. Lefranc acknowledges the support of the project Labex MME-DII (ANR11-LBX-0023-01). 


\section{Introduction}

Perhaps no other philosophical issue is as contentious as the debate over inequality and social justice. Most of the recent developments in the theory of justice start from the seminal intuition, discussed in Rawls (1971) that equality of individual outcomes is not necessarily fair. In order to determine whether inequality is legitimate or not, one needs to take into account the factors that determine observed outcomes. Responsibility-sensitive egalitarianism, whose prominent advocates in philosophy are Dworkin (1981a, 1981b), Arneson (1989) and Cohen (1989), and Roemer $(1993,1998)$ and Fleurbaey $(2008)$ in the economics literature, argues that individual responsibility represents the key criterion for assessing whether inequality should be seen as fair or unfair. This amounts to distinguishing between two sets of determinants of individual outcomes: circumstances include the determinants of outcome that lie beyond the realm of individual responsibility; effort gathers the determinants of which individuals are held responsible. Deciding what individuals should be held responsible for is of course a difficult issue that can be decided by each society on political grounds according to Roemer (1993). Once effort has been defined and observed, Equality of Opportunity (EOp) is usually considered to prevail if two conditions are met. First, individuals who exerted similar effort should face equal outcomes (compensation principle). Second, the impact of effort on the outcome should be respected somehow (reward principle).

Although equality of opportunity has been depicted as 'luck egalitarianism' by vocal critics (Anderson 1999, Hurley 2003) arguing that the "fundamental motivating aim of EOp is only to neutralize luck", the role of luck in the theory of equality of opportunity has largely been skimmed over in the economic literature. However, luck appears as a pervasive feature of everyday life, as emphasized in the recent essay of Robert Frank "Success and Luck" 1 In the commonsense view, luck is closely linked to randomness. It captures the unpredictable events that jointly determine outcomes once individuals have established their course of action, knowing their endowed assets (or lack thereof). Using the terminology of EOp theory, luck is deeply intertwined with circumstances and effort in the dynamic process of outcome formation. The goal of this paper is to offer a framework that allows to encompass luck in formulating the basic requirements of equality of opportunity, the compensation and reward principles, and to take into account the timing of the occurrence of luck.

Of course, luck is already partly incorporated in EOp theories, as circumstances can be viewed as

\footnotetext{
${ }^{1}$ Frank (2016).
} 
the luck of a birth lottery which allocates genes, families, and social environments to individuals. This birth lottery can be seen as an example of 'brute luck' which is randomly distributed across individuals throughout their life, and is often unobservable to third parties. Dworkin (1981a) is famous for having introduced the distinction between 'brute luck' and 'option luck', where option luck results from deliberate gambling choices. Dworkin suggests that no compensation is due to anyone who suffers a bad outcome due to option luck because individuals should be held responsible for their risk-taking. Fleurbaey (2008), however, challenges this view because the result of a lottery is clearly out of the responsibility sphere of an individual and claims that the effect of risk-taking should be erased in many cases. Even if they disagree on the treatment of option luck, both Dworkin and Fleurbaey admit that luck can be absorbed in the dual world of effort and circumstances.

On the other hand, Lefranc, Pistolesi \& Trannoy (2009) (henceforth LPT) believe that the project of separating the influence of random factors into circumstances and effort is too binary. They introduce 'residual luck' as a third determinant, and recommend something weaker than neutralizing residual luck. These authors are agnostic about what residual luck should precisely include and suggest that various ethical perspectives suggest different answers, although they point to a consensus that social background should be counted as a circumstance. These authors propose that a minimal requisite of EOp is that the residual luck should be equally distributed across types, at any given level of effort. This recommendation echoes Rawls's stance (1971, p.63) according to which "those who are at the same level of talent and ability, and have the same willingness to use them, should have the same prospects of success regardless of their initial place in the social system".

The present essay submits a more complete and deep discussion about how to incorporate luck into the definition of equality of opportunity. Our view is that the dual world of circumstances and effort may be too straight a jacket to encompass different views about luck and to fully account for the timing of luck. We maintain that a more flexible approach with respect to luck may have sense and that in addition to the two categories of effort and circumstances, it could be good to introduce one degree of freedom in the application of EOp principles, by distinguishing, from a normative point of view, a third set of determinants of outcome. In this paper, we call luck the third category which contains, if not all, at least some of the random factors that enter the determination of outcome and which, according to the ethical preferences of the social decision maker, should be treated differently from effort and circumstances. Both principles remain in substance. They are just redefined with luck having a neutral status in the 
reward and compensation principles: residual luck appear in the clause if and has a "neutral status".

This paper extends the analysis of luck of LPT in three important directions.

First, while LPT only focused on the compensation principle, this paper submits a new principle of reward based on the idea that effort deserves reward for the very fact it is costly. In so doing, we are moving away from the core concept of responsibility that is so pregnant in the equality of opportunity literature both in philosophy and economics. As a matter of fact, this principle of reward can be viewed as a minimal one since it only requires that the outcome function be increasing in a deterministic or probabilistic way. As such its incompatibility with the compensation principle, commented in the literature, vanishes. This contribution can be viewed as an extension of Roemer's proposals since he was the first to introduce the word "effort" in the semantics of equality of opportunity.

The second contribution lies in the explicit analysis of the timing of events (luck, effort and circumstances) and its implications for equality of opportunity. LPT only considered luck occurring before effort decisions were made. Here we also consider that good luck can be brought by higher effort. In that case, luck is revealed to the agent after effort has been exerted, without being revealed to third parties and it leads to the so-called moral hazard problem in contract theory. To the best or our knowledge, it is the first time that the problematic of moral hazard is introduced in the EOp setting. We argue that it leads to some intricacy that needs to be explicitly taken into account. In particular, moral hazard is different from betting because the action changes the subsequent random process, while decisions on the stake in betting does not. In both case, the drawing of a state of nature follows the action but in addition, in moral hazard the action influences the drawing in terms of a causal relationship with the important consequence that it makes less legitimate to neutralize the effect of luck on outcomes like in gambling.

The third contribution, allowed by the analysis of the time sequence of the determinants of outcome, is to discuss how the correlation of luck with effort and circumstances should be accounted for in the formulation of the compensation and reward principle. Should the correlation between luck and circumstances be viewed as a circumstance? Should the correlation between luck and effort be viewed as an effort? We show that various views can be taken leading to different formulations of the equality of opportunity requisite. Basically, two distinct formulations of the compensation and reward principles can be defined whose relevance depends on whether 
luck occurs before or after effort decisions are made. Each combination of principles correspond to a particular answer to the above questions about the status of the correlation of luck with effort or circumstances.

The rest of the paper is organized as follows. In section 2 we introduce the idea that effort deserves reward and propose an associated minimal reward principal in a certain world. We are then well equipped to present how the framework is modified by the introduction of luck in section 3 and in particular we discuss the idea that the timing of luck in the determination of outcome is important when understanding the relationship between luck, effort and circumstances. Next, in section 4 we formulate a first version of the compensation and reward principles in presence of luck from an ex-post view, i.e. EOp judgements are formulated at the end of the dynamic process that determines outcome, once all uncertainty has been resolved. In section 5 , we introduce a second version of the principles which correspond to an ex-ante view before effort is exercised and uncertainty is revealed. Section 6 offers a discussion of how these principles may be useful in thinking about different kinds of luck in relation with how the correlation of luck with circumstances and effort should be taken into consideration, as well as a general discussion of whether other principles than EOp should be appealed to when thinking about the role of luck in society. Section 7 concludes.

\section{Equality of opportunity without luck}

Before introducing luck in our model, it is worth setting the scene of our problem and discussing how it relates to standard features of the equality of opportunity literature. The equality of opportunity perspective rests on the seminal intuition that inequality of individual outcomes is not necessarily unfair: some factors should be compensated, yet others should be rewarded. We start by proposing that reward be associated to effort and not to choice. John Roemer coined the term effort but he did not interpret this word literally. Here we submit a literal interpretation of effort as an act inducing some cost for the agent. And for the very fact that it induces some cost, effort should somehow be rewarded. Next, we set up our model and finally we introduce our principle of reward in this context which along with the principle of compensation defines our theory of equality of opportunity in certainty. 


\subsection{Effort as deserving reward}

According to Arneson (2004), two distinct notions, which he refers to as Desert and Choice, can limit the scope of the egalitarian requisite. He defines and illustrates these notions as follows.

"Desert: The badness of inequality is lessened, the more it is the case that the relative level of good fortune that people reach is proportionate to their desert."

"Choice: The badness of inequality is lessened, the more it is the case that inequality arises via people's voluntary choices within a fair framework for interaction."

In the discussion of EOp, economists have strongly favoured the second notion, for reasons that are easy to understand. Modern economists have been heavily trained in microeconomics which is grounded in a theory of rational action. When Ronald Dworkin and Marc Fleurbaey endorse the view that people should be held responsible for their preference, they indeed stick to the famous latin maxim taken up by Gary Becker and Georges Stigler: "De gustibus non disputandum est". Gerald Cohen and John Roemer, as marxian, may be less comfortable with the notion of Choice and resort to a weaker version. Actions under the control of the agent may induce legitimate inequalities. Admittedly, it is difficult to defend that people are choosing some action if they are not controlling it. However, defining the scope of individual control in real life might be difficult without a kind of physiologic definition of what control is. Take the example of inmates in jail. Assume they are ordered down the prison's courtyard for a walk. They control their legs because their brain controls their legs. However, can we say that they control the action of walking in the courtyard? Likely not. Can we say that someone with the restless leg syndrome is controlling his legs? Probably not. The control view is really embedded in a physiological and psychological approach of the human behavior. The tenants of the control view have escaped providing a full description of this approach, which blurs what control really means in real life.

In this paper, we explore the other route: how do the notions of desert or merit place limits on the egalitarian requisite. We do not argue that it is ethically better than the Choice option but it can solve some problems that are met if we follow the Choice option. Obviously people may differ in their views over merit but, in everyday language, effort comes close to merit in many situations. Coming back to the jail example, suppose that inmates are assigned to two groups: the first group is requested to work part-time and the second one to work full-time and both groups are compensated for work. It would seem natural that the second group be 
more compensated than the first one, although inmates did not choose to belong to the first or second group. Our intuition is that we are inclined in that way because working is costly and for the very fact that it is costly, it should be rewarded 2 The dictum "The labourer is worthy of his hire" expresses this in common language. When Brian Barry argues against Roemer in the Asian student case, he resorts to a similar intuition (Roemer 1998 p.21): "Granted, he says, the Asian students have worked hard because of familial pressure, an aspect of the environment beyond their control, but nevertheless, if reward is due to effort, then they should receive more reward that the academic children, for they really tried harder". The prison's example is extreme but its walls symbolizes the social, economical, psychological constraints that hinder individual actions and choice. Economics simplifies a lot the description of human behavior to get a theory with positive implications that can be testified. But this simple description of the world does not count for all the constraints internal and external that individuals face and by the way it fails to provide a basis for normative statements in all situations.

As regard effort, we want to capture its main features in economic life which appear for instance in education (tertiary or vocational training), on the job market, in undertaking a business, saving decisions over the life cycle, or life styles choices in health care. If we come back to more common situations than the jail's example, it is convenient to take the example of the number of hours worked for a wage-earner working in a large firm within a regulated labor market like France. It is not fully convincing in all cases that the work duration is under the control of the agent or that it results from a free optimization, at least in the short run. In Savage's words, work duration will be an act but not a decision. The two popular views of preference or control then vanish in this case, whereas the cost argument can still be used to vindicate than someone who works in a firm in which the duration of work is 40 hours per week should be rewarded more than someone who works in a firm where he is constrained to work less 3 This cost interpretation of reward will be more convincing if there is a general agreement among individuals about the cost of effort. This is the route that we follow here.

We introduce our first set of determinants of individual outcomes, the set of individual efforts, which is possibly multidimensional and is denoted by $e \in \mathcal{E}^{p}$, where $\mathcal{E}^{p}$ needs not be a metric space. However, there is a quasi-ordering $\succeq$, not necessarily complete, defined on $\mathcal{E}^{p}$. $\succeq$ represents the agreement among individuals about how costly effort is, at least in ordinal terms. We

\footnotetext{
${ }^{2}$ Of course, the principle is meaningless if society does not value the outcome realized with effort as input. Thus in the background, the outcome introduced in subsection 2.2 below is assumed to be of value to all individuals and then to society.

${ }^{3}$ If work duration is statutory and differs across firms, it can be said that it is a circumstance. It should be compensated and in fact the cost of working more should be more compensated than the cost of working less. The principle of compensation can be useful if there is full cardinality and comparability of the cost of effort.
} 
interpret the fact that $e \succ e^{\prime}$ as meaning that all individuals agree that doing the multidimensional effort $e$ is more costly than performing the multidimensional effort $e^{\prime}$ for all individuals in a given society. This quasi-ordering respects the natural order of vector in $\mathbb{R}^{p}$ (at least one component strictly greater and no component strictly lower) and it captures a unanimous opinion across the population. For instance if we compare the multidimensional effort done by a surgeon during an operation and a teacher when lecturing assuming that both interventions last the same time, it is quite plausible that doing a surgical operation is universally viewed more painful than teaching. The assumption of the existence of a common partial ranking of effort levels can be stated (for economists) using a formal decision model where the utility function is specified in the simple quasi-linear form as a function of outcome $y$ (earnings) and effort

$$
u_{i}(y, e)=y-v_{i}(e)
$$

If we assume that $v_{i}($.$) is increasing and convex, then the quasi-ordering will be given by the$ intersection of all orderings represented by the $v_{i}($.$) . The fact that the individuals interpret the$ effort levels in an unanimous way makes the support of the reward principle stronger. But we insist that our vision of effort does not require that it has been the result of an optimization process.

Ultimately, this common view about effort which allows people to rank effort in a universal way, even if it is likely a partial ranking, should be tested in lab experiments. Another direction would to consider progress made by neuroscientists about measuring physical pain and discomfort. Pain scales have been used for some time in the treatment of various diseases. A step has been made by measuring thermal pain in a controlled lab experiment $]^{4}$ Cerebral circuitry is far from having revealed all its secrets, but we can hope for major progress in this century.

\subsection{Analytical framework}

The analytical framework requires the definition of two more sets, the set of outcomes and the set of circumstances.

We assume that individual outcome can be measured by a positive scalar $y$. Two main qualifications are important with respect to how the outcome is defined in the literature.

First, we focus on the final outcome enjoyed by individuals in the population, after any possible

\footnotetext{
${ }^{4}$ See for instance Brown, Chatterjee, Younger \& Mackey (2011) and Wager, Atlas, Lindquist, Roy, Woo \& Kross (2013).
} 
redistributive policy. Our objective is limited in the sense that we are only willing to set up a model for characterizing a fair distribution of outcome $y$ in the population, from the perspective of EOp. Contrary to Roemer (1998) and Fleurbaey (2008), we do not seek to define optimal policies from the point of view of EOp, given an unfair allocation. In fact, in several instances, the distinction between the distributions of outcome before and after public policy is difficult to draw and not particularly meaningful. For instance, it is not possible to observe individual health before public intervention. Even in the case of income, public education is likely the most powerful instrument to weaken the impact of circumstances, although it is hardly possible to estimate pre-education income.

Second, we focus on gross outcome in the sense that the cost of effort has not been netted out (see the utility function introduced previously). In the simplest case, the net outcome would be given by the expression (1) for all individuals. However, unless we suppose interpersonal cardinal comparison of the cost of effort (the $v_{i}($.$) are the same up to the same affine transformation)$ we cannot compare the outcome net of the cost of effort across the population. The focus on gross outcome is therefore motivated by the fact that it is precisely the only variable in the population that can be steered by the public policy.

The second set of determinants comprises individuals circumstances, denoted by $c \in \mathcal{C}^{n}$, where $\mathcal{C}^{n}$ is a space of dimension $n$ which is not necessarily a metric or an ordered space. This set gathers all the determinants of individual outcomes that are not considered as legitimate sources of inequality across individuals. As such, their effect on the distribution of outcomes should be nullified. We refer to a type as the set of individuals with similar circumstances.

The determination of outcome can be summarized by the outcome function $Y$ relating individual outcome $y$ to individual circumstances $c$ and effort $e$ :

$$
\begin{aligned}
\mathrm{Y}: \mathcal{C}^{n} \times \mathcal{E}^{p} & \rightarrow \mathbb{R}^{+} \\
(c, e) & \rightarrow y=\mathrm{Y}(c, e)
\end{aligned}
$$

The outcome or reward function is a reduced form function that is influenced the public policy implemented in the society. Knowledge of this function is thus not sufficient to choose a particular policy that would optimize some social welfare function. 


\subsection{EOp principles without luck}

As discussed by several authors, the requisite of equality of opportunity can be characterized by two principles: the compensation principle and the reward principle 5 Here both principles are defined in terms of properties that the function $Y$ should satisfy.

The minimal reward principle: effort should pay Since effort is the only source of legitimate inequality in this model, exerting a greater effort may go along with a higher outcome. The EOp ethics goes beyond this mere chance of a better life. It transforms an option into an ethical requirement. Individuals exerting greater effort must enjoy a higher level of outcome. The reward principle should be defined in an independent way of the compensation principle. We cannot then preclude that the compensation principle is not implemented in the definition of the reward principle. The inter-individual comparison of effort should only apply to individuals within type, i.e. given the same level of circumstances $c$.

Definition 1 (Reward principle without luck) The reward principle is satisfied iff for any $c \in C^{n}$, for any $e, e^{\prime} \in \mathcal{E}^{p}$, such that $e>e^{\prime}$ (at least one component strictly larger),

$$
\mathrm{Y}(c, e)>\mathrm{Y}\left(c, e^{\prime}\right)
$$

The formulation of this principle appears to be minimal in only requiring that the outcome function be strictly increasing in effort. Observe that this principle, albeit minimal, requires that people are able to rank efforts. If two efforts are non-comparable, the principle vanishes.

This formulation of the reward principle can be compared to other principles found in the EOp literature. The request of increasingness has already be formulated by Cappelen \& Tungodden (2006) in an even weaker form: there should exists a social state in which a person exercising high effort gets a higher post-tax income than a person with the same talent exercising low effort.

Roemer does not include as an explicit requisite of EOp that effort should pay. He almost exclusively emphasizes the principle of equality of outcomes for a given effort, or if not possible, the second best solution that maximizes the outcome of the worst-off. Fleurbaey has however showed that Roemer implicitly appeals to a principle of reward. Fleurbaey coined the term of utilitarian reward principle to say that the redistribution of resources among individuals within

\footnotetext{
${ }^{5}$ See for instance Fleurbaey (2008), or Ramos \& Van de gaer (2016) for a recent survey
} 
a type arises from the maximization of a Benthamite social welfare function, something that Roemer recommends. Since utilitarianism corresponds to a zero-inequality aversion from the social planner, the pre-tax inequality among a type is not reduced except in specific cases, and if the effort pays before tax, it would go on after tax. And indeed, Roemer assumes that the outcome function before state intervention is monotone increasing in individual effort, although this is mostly seen as an identification condition, i.e. a way to infer individual effort, conditional on outcomes and circumstances. All in all, one can consider that Roemer's solution satisfy our minimal reward principle, even if it is not explicitly stated as such.

Fleurbaey (2008) discusses extensively the reward principle as being part of the equality of opportunity requisite. The framework of analysis is a discussion of public policy. In this context, the reward principle requires that compensation does not alter the natural reward of effort observed before policy intervention. In a nutshell it amounts to requiring that the increase in outcome associated with a rise in effort be unchanged after policy intervention. While we require that effort pays, we remain silent on the issue of how much effort should pay. In particular, we do not require that it pay more or less than in the "free" market situation. Our principle is compatible with effort paying more than in the "free" market, as would be case for the working poor under a tax credit system such as the US EITC. Hence our formulation appears as a minimal reward requisite.

The compensation principle: circumstances should be irrelevant One of the key insights of equality of opportunity theories is that inequality arising from differential circumstances is morally offensive: fairness demands that individuals face similar outcomes, regardless of their circumstances. However, one has to be more specific because differences in effort are viewed as a source of legitimate inequality. Hence, the requirement of equality of opportunity should only apply among individuals with similar $e$. To summarize, circumstances define what should be compensated and effort defines the scope for relevant inter-individual comparisons. If all the determinants of outcomes belong to either $c$ or $e$, the compensation principle can be formulated as follows:

Definition 2 (Compensation principle without luck) The compensation principle is satisfied iff for any $e \in \mathcal{E}^{p}$, for any $c, c^{\prime} \in C^{m}$,

$$
\mathrm{Y}(c, e)=\mathrm{Y}\left(c^{\prime}, e\right)
$$


Figure 1: Outcomes, effort and circumstances

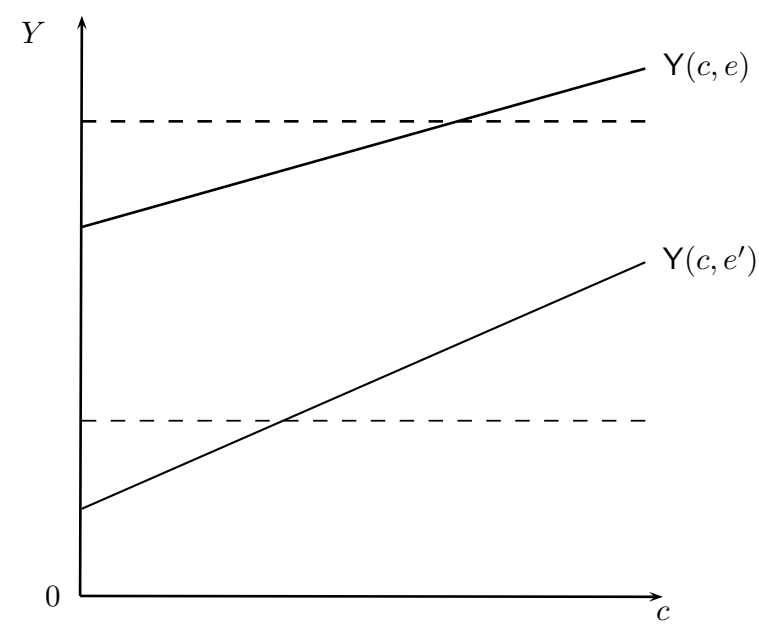

This is what Fleurbaey (2008) refers to as equal outcome for equal responsibility.

In the end, the combination of our two principles can be illustrated by the following Figure borrowed from Fleurbaey \& Schokkaert (2009). Individual outcome is represented on the vertical axis and circumstances are assumed to be captured by a positive scalar represented on the horizontal axis. The two increasing lines represent individual outcome as a function of circumstances $c$, for two levels of effort, $e$ and $e^{\prime}$, with $e>e^{\prime}$. Obviously, in this case, individual with greater values of $c$ are advantaged. Conditional on $c$, outcome increases with $e$.

Our conditions require that conditional on effort, the gradient of outcome w.r.t the circumstances be nullified (compensation principle) and that the outcome lines be ranked by order of effort level (minimal reward principle). This situation is satisfied by the two dashed lines. Contrary to Fleurbaey, we do not require, as in the natural reward principle, that the differential reward associated with increased effort be equal to its observed value before policy intervention. In fact, in the case of this Figure (and more generally whenever the cross-derivative of outcome w.r.t effort and circumstances is not zero), this cannot be uniquely defined and requires to single out a particular level of circumstances.

\section{Luck and its timing}

We enrich the model by introducing uncertainty and we discuss how luck may interact with effort and circumstances in a dynamic setting, in the determination of outcome. 
In ordinary conceptions, luck is closely linked to the notion of randomness. In general, luck is understood as the chance happening of fortunate or adverse events. It would thus usually refer to the determinants of outcome that result from random processes. For instance GarciaGomez, Schokkaert, Van Ourti \& Bago d'Uva (n.d.) define luck as "everything which could not be predicted (ex ante)". Obviously, randomness plays a role in individual outcomes: who you met in life, whether you were endowed with a particular gene, how lucky you were on the day of the exam... are important determinants of individual success that at some point or another should be seen as random draws.

Given the prevalence of these random factors in the determination of individual outcome, it is worth investigating how they can be accommodated in the definition of equality of opportunity. Let us take as our starting point the model of the previous section where outcome is assumed to be fully determined by some circumstances $c \in \mathcal{C}^{n}$ and some effort $e \in \mathcal{E}^{p}$. Now assume further that beyond the determinants captured by $c$ and $e$, there exists some additional random factors denoted $\omega \in \Omega^{q}$, where $\Omega^{q}$ is not necessarily a metric or an ordered space 6 These random factors are identical in essence to the states of nature in decision theory. Ex post, only one state of nature is be revealed to the individual. However, ex ante, he does not know which state of nature will occur. This leads to redefine $Y$ the function relating individual outcome to its determinants $c, \omega$ and $e$ as:

$$
\begin{aligned}
\mathrm{Y}: \mathcal{C}^{n} \times \mathcal{E}^{p} \times \Omega^{q} & \rightarrow \mathbb{R} \\
(c, e, \omega) & \rightarrow y=\mathrm{Y}(c, e, \omega)
\end{aligned}
$$

The key question from an equality of opportunity perspective is how the random factors captured by $\omega$ should be accounted for in the principles of compensation and reward. According to some authors, these factors should count as circumstances (Fleurbaey) or as effort (Roemer). Our main proposal is that some of the components of $\Omega^{q}$ cannot be reduced, from a normative perspective to the moral categories of circumstances and effort. Most of the random factors differ from the notion of effort discussed in the previous section in the sense that the occurrence of luck cannot be directly traced to some costly action undertaken by the individual, although than can at the same time be influenced by individual decisions. These random factors also differ from circumstances in the sense that they cannot be predicted, while for most individual, major circumstances such as family and social background are largely known at the age of

\footnotetext{
${ }^{6}$ We will later signal the rare instances where the assumption of a metric space is required.
} 
consent when individuals make effort decisions.

We define luck as the residual part of the random factors in $\omega$ that cannot be ascribed to the two previous categories. We denote this third set of determinants by $l \in \mathcal{L}^{r} \subset \Omega^{q}$ with $r \leq q$. Using this notation, we can thus redefine the outcome function $Y$ as:

$$
\begin{aligned}
\mathrm{Y}: \mathcal{C}^{n^{\prime}} \times \mathcal{E}^{p^{\prime}} \times \mathcal{L}^{r} & \rightarrow \mathbb{R} \\
(c, e, l) & \rightarrow y=\mathrm{Y}(c, e, l)
\end{aligned}
$$

where the dimensions of the sets of circumstances and effort has been adjusted to reflect the fact that they now incorporate part of the dimensions of $\left.\Omega^{q}\right]^{7}$

The reasons that lead to single out some of the random factors in $\omega$ as deserving a special treatment will be discussed further in the next two sections, where we redefine the EOp principles to account for luck. To a large extent, these reasons relate to the dynamic interplay of circumstances, effort and luck in the determination of outcome, as we now discuss.

A full account of the intricacy of effort, circumstances and random factors would require a proper dynamic model of the outcome determination process. Such a model would allow to describe the interaction between compensation-deserving and reward-deserving factors and to identify the key stages in the formation of inequality, both legitimate and illegitimate. In fact, a positive dynamic model of outcome formation seems to be a key condition for the design of optimal compensation policies, as emphasized for instance in Cunha \& Heckman (2007). The focus of our paper is different and concentrates on the characterization of equality of opportunity in the distribution of final outcome. Hence, we focus on the resulting effect of the various factors on individual final outcome, and we leave for future research the analysis of the dynamics of inequality formation.

However, it is important to keep in mind that these random factors can intervene at different stages of the formation of outcome. In fact, from the perspective of EOp, they should be distinguished according to the stage at which they occur. In particular, random factors occurring before effort decisions are made should be distinguished from those occurring after effort. This is the key idea behind classical decision theory, as expressed for example by Savage (1954), which analyzes how subsequent uncertainty affects individual decision. To a large extent, our representation is close to that of decision theory and views randomness before effort choice as

\footnotetext{
${ }^{7}$ In the rest, for notational simplicity, we omit this adjustment.
} 


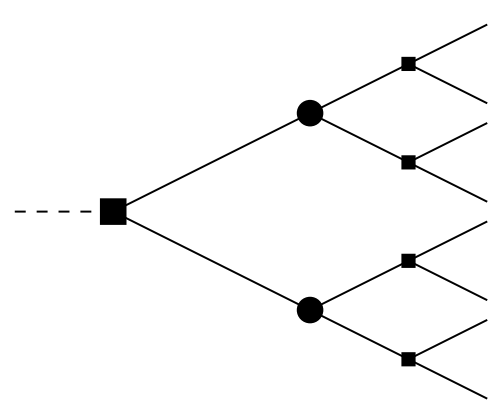

being part of circumstances and residual randomness occurring after effort choice as the bulk of the luck category.

Figure 2 provides a first illustration of the interplay between circumstances, effort and random factors in the determination of individual outcomes. Outcome formation is depicted by a decision tree under uncertainty where the individual plays against nature. End nodes are assigned outcome levels in $\mathbb{R}_{+}$. Decision nodes are indicated by a square and chance nodes are represented by a circle. The starting gate at the age of consent (the first node not represented) captures individual background (genes, family and social context).

This figure illustrates the intricacy of random factors, circumstances and effort. First, it is worth emphasizing that the final outcome is jointly determined, at the different stages of the decision tree, by the combination of all three factors. Random factors do not act in isolation from the joint effect of individual circumstances and effort. In particular, random factors are intertwined with individual decisions and can occur at different stages, either before or after individual decisions have been made. Of course, the random factors in the last stage may in some cases operate in a way that is independent of other determinants: for instance, a white noise shock may affect the level of outcome determined by $c$ and $e$. However, it may be more natural to think that the distribution of random shocks operating at this stage varies along the different paths of the decision tree. In other words, using the terminology of decision theory, the distribution of states of nature may be influenced by individual choices. The term of moral hazard has been coined to capture the phenomena where choosing a higher level of effort may bring better draws of these random factors. In others words, choosing an effort level is associated with a specific random draw.

To some extent, random factors could also occur before effort decisions are made. In fact, 
Figure 3: The timeline: different types of luck

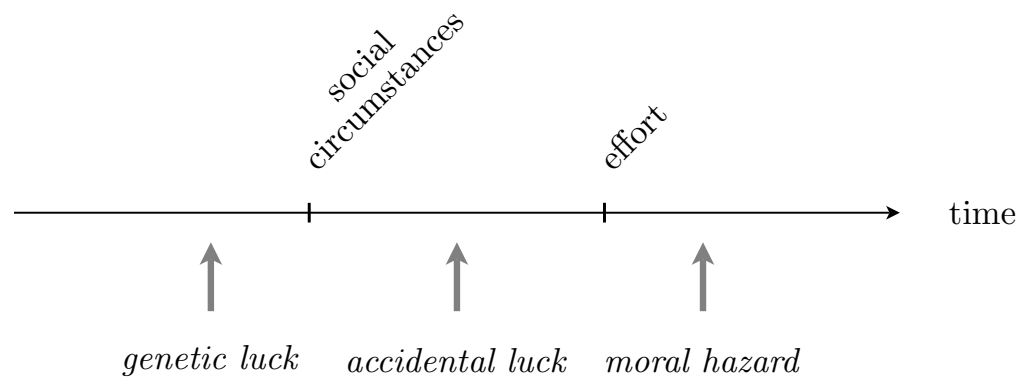

circumstances represented by the dashed line feature this idea. Of course, from the starting gate position of adult life, individual circumstances are to a large extent already known and are not uncertain. However, from the perspective of the original position, these factors can be seen as the result of a gigantic lottery, the birth lottery. Figure 2 also illustrates the possible influence of circumstances on both effort and subsequent random factors. The influence of circumstances on individual effort has already been developed in LPT. But it is also worth emphasizing the fact that the influence of circumstances on final outcomes may not be fully deterministic. It can also be mediated by the occurrence of random factors as discussed in a companion paper (Lefranc \& Trannoy 2017).

In the following, we will keep in mind the above decision tree in the simple form of a timeline presented in Figure 3 . When taking his decisions, the individual will likely know the circumstances, that is, all the events that happen before the age of consent are revealed to him. Think of family and social background and some biological traits, as beauty, height, some ability to learn and memory for instance. On the one hand, circumstances occur first and are known when the individual is taking decisions. On the other hand, the impact of effort on outcome is mediated by random factors in a way that the individual cannot fully predicted. Even if for the social observer innate talent and social background can be viewed as random, the fact that they are antecedent to the decision process suggests that they be considered as circumstances. Indeed, antecedent random factors determine the course of actions taken by the individual but are not determined by it. In the case of moral hazard the causality runs in the opposite direction: decisions can impact future random factors.

Finally, let us introduce two additional refinements in the case of random factors occurring before effort. In a simple view, all such factors should be treated as circumstances. However, it might be important from a normative viewpoint, for reasons that would appear more clearly in 
the following sections, to distinguish among them. A first category comes to mind, biological traits or genetic luck that can be thought to occur even before, let us say, social circumstances, the social and family background where you have been grown up. Second, there might be some random draws occurring after these social circumstances that may or may not be associated to them, for instance some diseases. They will be referred to as accidental luck. As argued in the following section, both genetic and accidental luck should somehow be distinguished from circumstances in the definition of equality of opportunity.

We are now well equipped to present two views about how to define the compensation and reward principles with luck. In the first view, we are approaching the problem when all uncertainty has been resolved, at the end of the process of outcome determination, i.e. at some end point of the tree. We call it the ex-post view (Section 4). In the second view we assess the allocation of outcomes just before the individual is exerting some level of effort, in particular before moral hazard unfolds. We refer to this view as the ex-ante view (Section 5). It turns out that the different sorts of luck that we have singled out are not treated in the same way by these two perspectives.

\section{The ex-post view: point-wise principles}

The objective of this section, as well as the next one, is to formalize the compensation and reward principles in the presence of luck. In the present section, we take on an ex-post perspective when evaluating the distribution of outcomes. This amounts to assuming that all information on the determinants of outcome have been disclosed to the social observer. We first formulate the two principles in this setting. We then explain by means of examples how luck may be isolated from a pure normative point of view.

\subsection{Point-wise principles}

The compensation principle The compensation principle states that individuals should receive similar outcomes regardless of their circumstances, other things being held equal. In the dual world where determinants of outcomes belong either to circumstances or effort, these other things simply denote effort, which leads to the conditional equality requirement discussed in the previous section. This key intuition remains in the presence of luck, and luck is now included among the set of conditioning variables. In fact, one has to decide whether the effect 
of luck should be fully preserved or whether differences in luck that are correlated to individual circumstances should also be nullified. This results in two distinct conditional equality principles of which only the first one is presented in this section. The point-wise view requires that the effect of luck on outcome be fully preserved. In this case, the principle of compensation requires that individuals with similar effort and similar luck should enjoy similar outcomes. This leads to the point-wise compensation principle, which we state as follows:

Definition 1 The point-wise compensation principle $(P C)$ is satisfied iff : for any $\left(c, c^{\prime}\right) \in$ $\mathcal{C}^{n} \times \mathcal{C}^{n}$, for any $l \in \mathcal{L}^{r}$, for any $e \in \mathcal{E}^{p},$,

$$
\mathrm{Y}(c, e, l)=\mathrm{Y}\left(c^{\prime}, e, l\right)
$$

The issue of the correlation between luck and circumstances is escaped in the point-wise formulation of the principe of compensation. The positive or negative correlation between the random variable $l$ and circumstances will remain unaffected. Note that luck goes along with effort as a conditioning variable. It seems as it is considered as an effort variable but this confusion will be clarified by stating the point-wise reward principle.

The minimal reward principle Extending the reward principle to account for the presence of luck, follows the same logic. It amounts to requiring that effort increases outcome, holding constant the value of the other determinants of outcome, i.e. circumstances and luck in this case. Again, the question arises whether the correlation between effort and luck should be rewarded or not. The point-wise reward principle assumes that the correlation between effort and luck should be treated as luck. This leads to the following formulation:

Definition 2 The point-wise reward principle $(P R)$ is satisfied iff: for any $c \in \mathcal{C}^{n}$, for any $l \in \mathcal{L}^{r}$, for any $e, e^{\prime} \in \mathcal{E}^{p}$, such that $e>e^{\prime}$ (at least one component strictly larger),

$$
\mathrm{Y}(e, c, l) \geq \mathrm{Y}\left(e^{\prime}, c, l\right)
$$

This is similar to the formulation of the reward principle in the previous section, where luck sides along with circumstances as a conditioning variable. It amounts to requiring that effort pays, given the revealed value of luck. Hence, it seems better suited to characterize situations where luck is antecedent and exogenous with respect to the choice of effort. 


\subsection{Why single out luck ?}

Overall, the comparison of the point-wise principles with their formulation in the absence of luck reveals that luck holds an intermediate position between circumstances and effort. It appears as a conditioning variable in both principles, meaning it is treated in the same way as effort from the perspective of compensation (i.e. it does not require compensation) and in the same way as circumstances from the perspective of reward (i.e it does not require reward).

We now examine why some of the random factors affecting individual outcome and discussed in section 3 should be distinguished from the two normative categories of circumstances and effort. In this section, we develop two main arguments. The first one pertains to the treatment of deliberate gambles. The second one pertains to the partial and asymmetric compensation for genetic luck.

Deliberate gambles: responses to Dworkin, Fleurbaey and Le Grand We illustrate the application of our extended compensation and reward principles to the case of deliberate gambles. The outcome of deliberate gambles can be seen as the product of two factors: on the one hand, the gambling decision per se (how much an individual bets on a particular lottery number) which should be treated as effort; on the other hand, the random draw of the gambling outcome (whether you chose the winning number), which should be treated as luck. Decisions on the stake should not only be removed from circumstances but should be rewarded in the sense that they are costly to the agent. Including the stake as part of effort amounts, according to the reward principle, to require that among individuals who bet on similar numbers, those who incurred a higher cost, in the form of higher-stake bets, should be rewarded more. On the other hand, the random process that decides on the winners should be neither compensated nor rewarded and is included as luck in both principles.

We now discuss how this compares to other solutions proposed in the literature. Three prominent views have been expressed by Dworkin, Le Grand and Fleurbaey. Our solution is in line with Dworkin as far as compensation is concerned but may differ in terms of reward.

Dworkin (1981a) introduced the now famous distinction between 'brute luck' and 'option luck'. According to him, "Option luck is a matter of how deliberate and calculated gambles turn out whether someone gains or loses through accepting an isolated risk he or she should have anticipated and might have declined" (Dworkin 2000, 73). Brute luck is "a matter of how risks fall out that are not in that sense deliberate gambles" (Dworkin 2000, 73). Dworkin's view is that 
no compensation is due to anyone who suffers a bad outcome due to option luck because individuals should be held responsible for their risk-taking decisions. This suggests that option luck be excluded from the set of circumstances, a position that our proposal shares with him. This implies that the compensation principle does not apply to the results of option luck. Furthermore, in the binary world where outcome determinants are partitioned into circumstances and effort, option luck would, according to Dworkin, count as effort. Hence, the libertarian reward principle should apply to option luck. This latter requirement can obviously be challenged on the grounds that once the gambling decision has been taken, the randomness in outcome escapes individual merit. This suggests that this pure randomness should be excluded from the scope of application of our reward principle. In the case of gambling, there is no intrinsic merit to having drawn a winning ticket at the lottery. In fact, this is precisely the meaning of definition 2, One should emphasize that contrary to Dworkin, our minimal reward principle simply requires that lucky high-stake gamblers receive more than equally lucky low-stake gamblers but leaves open the possibility of partial redistribution of the gains. This option is not open according to the libertarian reward principle.

Fleurbaey (2008) first recalls the solution of Le Grand (1991), which challenges this approach. He suggests that the effect of deliberate gambles be split into two parts: the decision to take the gamble, and the random draw. This amounts to treating the risk-taking preference of the individual as a responsibility characteristic, and, conditional on the decision taken, to consider the outcome of the gamble as a circumstance, which should be nullified. In other terms, individuals are fully insured against residual risk, conditional on their stake decisions. However, this may violate individual decisions to deliberately enter gambles and diminish the welfare arising from such decisions 8

The opportunity to engage into decisions that yield uncertain outcomes might, in some cases, enhance individual well-being, not only ex-ante but also ex-post. Bets and lotteries represent in developed societies a sizable share of individual resources. For instance, in France, aggregate (legal) bets amount, in 2012, to 32 Billions euros or $2 \%$ of national income. People's decision to enter typical lotteries might at first sight be difficult to explain without assuming risk-loving preferences. In general these lotteries are characterized by a relatively low-payout rate and (very) small odds of winning. However, some authors have noted that existing lotteries are often characterized by skewed-payoff distributions: losses are typically frequent and small and gains

\footnotetext{
${ }^{8}$ Some may contend that the pure pleasure of gambling remains intact even if outcomes are equalized ex post. However, casual empiricism suggests that the thrill of poker games is higher when monetary gains are at stake, compared to high-school games where the only stake is a handful of matchsticks.
} 
are usually very large and rare. If individuals exhibit preference for right-skewed lotteries, even risk-averse individuals might prefer to enter lotteries (see Golec \& Tamarkin 1998, Chiu 2010). Fleurbaey further elaborates on Le Grand to offer a more sophisticated solution. He notes that there might be a conflict, when evaluating the impact of lotteries on individual welfare, between the ex-ante and ex-post perspectives. Most gamblers might be better off gambling ex-ante, in terms of expected welfare, and at the same time be worse off ex-post, once the dice has been rolled and they end up losing. In an egalitarian perspective, this might be an issue. To solve it, one needs to decide whether ex-ante or ex-post preferences should be taken into account when evaluating gambling decisions. Fleurbaey (2008) argues that one should give priority to ex-post preferences since individuals are better informed ex-post about their preferences. He takes into account informed preferences, that is, preferences that the individual discover after the true state of nature. As a matter of example, Fleurbaey (2008 p.162) distinguishes between risk lovers and super risk lovers. If they lose, the former regret gambling ex-post whereas the latter do not. Following the distinction introduced by Kahneman, Wakker \& Sarin (1997) between decision utility and experience utility, preferences that might be respected are 'experience' preferences, in the present case, the preferences of the super risk lover. Fleurbaey does not propose a compensation scheme for them and therefore leaves room for uninsured risky activities. However, people who regret their gamble will be compensated as in the Le Grand solution.

Empirically, it is possible to examine the social preferences for redistribution of the gains of gambling decisions by looking at the fiscal treatment of these gains. In France for instance, gains from the national lottery are exempted from taxation. This seems to indicate that gains from lotteries are fair, which is consistent with the ex-ante evaluation of gambling decisions or an ex-post view point if all losers are super risk lovers $9^{9}$

Partial and asymmetric compensation of genetic luck A second argument for considering a third generic class of variables, in the definition of equality of opportunity lies in actual preferences for redistribution. There are cases in which the type of redistribution favored by individuals seems to call for only a partial and asymmetric compensation of factors that nevertheless lie beyond the realm of individual responsibility.

Take for instance the case of genetic endowments. This represents a source of inequality that

\footnotetext{
${ }^{9}$ Of course, one may contend that the tax treatment of gambling gains does not simply reflect collective preference for redistribution but also reflects the government's objective to provide incentives to participate in revenue-generating gambling.
} 
obviously lie beyond individual choice. Hence, a strict application of the criterion of individual responsibility would command that this source of inequality be fully neutralized. On the contrary, the standard libertarian argument would claim that genetic endowments are a fully legitimate source of inequality, since they are constitutive of the individual. Hence, genetic endowments would count as effort and not be compensated, which can also be taken into account in the dual world.

In many cases, however, individual conceptions of fairness might stand in a intermediate position between these two alternatives, and blend together the libertarian self-ownership arguments and the egalitarian responsibility perspectives (see Vallentyne 1997). For instance, people might want to compensate the effect of poor genetic endowments and at the same time leave individuals free to enjoy the benefit of a particularly favorable genetic luck. An example could be a situation in which, one supports the compensation of muscular dystrophia and at the same time let people enjoy the benefits of a particularly good health constitution. Similarly, some individuals might at the same time advocate for the compensation of unfavorable cognitive skills endowments, say through special education programs and support at the same time the possibility for individuals with special talents to develop them and enjoy the benefits thereof 10

These composite redistributive principles are hard to fit into the dual world. And this could motivate the introduction of a third category, luck, in a comprehensive model of compensation and reward. One possibility of forcing asymmetric compensation would be to split genetic luck into two components: the bottom of the distribution which would count as circumstances and be compensated and the top of the distribution which would be treated as luck in both principles. This would imply that having a good genetic endowment would be left uncompensated by the point-wise compensation principle. At the same time, the reward principle would not apply to good genetic endowment, for instance in the health context.

\section{The ex-ante view: the distribution-wise principles}

In the previous section we adopted an ex-post perspective, in the sense that equality of opportunity was assessed once all uncertainty had been resolved. Alternatively, one can endorse an ex-ante point of view, and assess equality of opportunity before the individual knows the state of nature that will prevail (for a discussion about ex-post and ex-ante see Fleurbaey (2010)).

\footnotetext{
${ }^{10}$ In fact, such positions might echo the previous discussion on the preference for skewness in gambling contexts: the asymmetric compensation for luck would lead to a skewed distribution of the consequences of genetic luck that would be welfare enhancing, from the ex-ante perspective where genetic endowments have not been drawn.
} 
This amounts to characterizing equality of opportunity from the perspective of the starting gate position where individuals make their effort decisions, before all subsequent uncertainty is resolved. We first argue that the ex-ante view is particularly needed to cope with moral hazard. We then state an ex-ante view of the compensation and reward principles.

\subsection{The distinctive features of moral hazard}

As already discussed, moral hazard designates the random factors drawn after effort choice has been exercised and that are correlated with effort. Even if a good deal of the discussion in the literature about luck has been focused on gambling, gambling per se is far less ubiquitous than moral hazard. Let us come back to Figure 3 in which individuals choose their effort level. In this ex-ante perspective, some individual circumstances are known. Yet other factors are not fully determined and are ex-ante uncertain. They can be considered as random and their occurrence lies beyond the realm of individual responsibility. However, to some extent, the random distribution of these unknown factors is not independent from the choice of effort. In particular, choosing a higher level of effort may bring better draws of these random factors, in various ways. For instance, the distribution of random factors attached to higher effort might be better, in the sense of first-order stochastic dominance: as an example, the distribution of exam grades for a student working harder might dominate the distribution of grades he would have if he studied less. In other cases, the benefits of effort might take the form of a safer outcome distribution, in the sense of second-order stochastic dominance, as in the case of voluntary and costly prevention. These forms of correlation between effort and random factors are probably widespread. Educational or job search decisions, to name just a few, are key responsibility variables of which the consequences are not deterministic. Hence, it seems important to account, in the definition of equality of opportunity, for the correlation between effort and subsequent random factors.

This correlation raises interesting issues for the definition of equality of opportunity. If effort is a legitimate source of inequality, it seems natural that respecting the effect of effort on outcomes also requires respecting the consequences of effort in terms of more favorable luck distribution. This should be reflected in the principle of reward. Let us observe that the point-wise reward principle is useless to this respect since it gets rid of the correlation between effort and luck. This went unnoticed when we introduced the point-wise reward principle for the very reason that in the case of deliberate gambling, this issue is indeed irrelevant. In fact, in a pure gambling 
context, the action of gambling has no influence of the distribution of luck, contrary to the setting of moral hazard. The ex-ante perspective allows to take this correlation into account. At the same time, luck remains distinct from effort, as luck is not per se a source of individual merit and the principe of reward should not apply to luck as it applies to effort. It seems clear that the principles of reward should be redefined to account for moral hazard. It is the aim of the following subsection.

\subsection{Distribution-wise principles}

Individual realization of outcomes, circumstances, effort and luck, are still denoted respectively by $y, c, e$ and $l$. Each of these variables can be seen as randomly distributed in the population. We use upper case letters $Y, C, E$ and $L$ to denote the corresponding random variables in the population. $f_{X}$ denotes the distribution function of variable $X$ in the population and $F_{X}$ denotes the associated cumulative distribution function. Lastly, we denote by $f_{X \mid Z}$ the distribution in the population of variable $X$ conditional on variable $Z{ }^{11}$ The ex-ante perspective defines the requisite of equality of opportunity in terms of these distributions, which vindicates that we can name them the distribution-wise principles.

Two remarks are in order to better understand the meaning of the ex-ante approach. First, it is quite implausible that the distribution of luck is known ex-ante. In most cases, the major source of information about the distribution of luck is obtained once all the uncertainty is resolved. The ex-ante perspective is then based on ex-post data. The frequency approach of probability will be useful to build an empirical CDF of luck. Second, it is important to realize that the only factor that is not fixed when computing the CDF of outcome conditional on effort and circumstances is luck. Namely the distribution of luck is what makes the distribution of outcome non-degenerate, i.e. distinct from a single mass-point distribution, given the value of circumstances and effort. Mathematically, define $\mathcal{L}(y, c, e)=\left\{l \in \mathcal{L}^{r} \mid \mathrm{Y}(c, e, l) \leq y\right\}$. Then by definition,

$$
F_{Y \mid C, E}(y \mid c, e)=\int_{l \in \mathcal{L}(y, c, e)} f_{L \mid C, E}(l \mid c, e) d l .
$$

We first examine the minimal reward principle and next turn to the compensation principle.

\footnotetext{
${ }^{11}$ Strictly speaking, introducing distribution functions and cumulative distribution functions, as we do here, presupposes that the set $\mathcal{L}^{r}$ be a metric space. We make this assumption for ease of notations. However, the results derived below could generalize easily to the case where $\mathcal{L}^{r}$ is not a metric space by using dirac mass distributions on the universe of luck.
} 
The distribution-wise reward principal As previously discussed, the minimal reward principle requires that effort should pay. In the previous section, we required that the outcome function be increasing in effort, given circumstances. This is consistent with the view that circumstances and luck are exogenously given and that effort choice is made knowing circumstances and luck.

However, as previously discussed, there might be cases where part of the payoff to increased effort is to bring more favorable luck. In this case, conditioning on luck will absorb part of the benefit of effort. To allow for the fact that the benefit of higher effort might take the form of better luck, one might condition only on circumstances when defining the principal of minimal reward. Hence, we may want to impose that effort pays in terms of the overall distribution from which individuals draw their outcome but not necessarily for each degree of luck. This leads to a second formulation of the reward principle that we define as the distribution-wise reward principal (DR).

Definition 3 The distribution-wise reward principle (DR) is satisfied iff: for any $c \in \mathcal{C}^{n}$, for any $e, e^{\prime} \in \mathcal{E}^{p}$, such that $e>e^{\prime}$ (at least one component strictly larger), we have:

$$
F_{Y \mid C, E}(\mid c, e) \succeq_{F S D} F_{Y \mid C, E}\left(\mid c, e^{\prime}\right)
$$

where $\succeq_{F S D}$ denotes first-order stochastic dominance

The perspective behind the DR principle can be seen as an ex-ante point of view where effort is chosen before luck is determined and potentially influences the luck draw. This justifies why, in this perspective, individual outcomes need to be evaluated on the basis of the distribution individuals face. Since uncertainty is an integral part of this distribution and individuals cannot be assumed to be risk neutral, the outcome prospects cannot be rightfully summarized by the expected (mean) outcome and the full distribution must be considered. Of course, this makes the formulation of the reward principle more complicated. To translate the idea that effort should pay, one may require that the distribution of individual outcome be strictly better for greater levels of effort, in the sense of first-order stochastic dominance. This may appear to be too restrictive and would not allow to fully account for the relationship between effort and luck. For instance, as discussed above, effort might take the form of greater prudence exerted to access a safer distribution. In this case, it might be thought that requiring first-order dominance might be excessive. However, we claim that because $y$ is the gross outcome and not the outcome net of cost of effort, the distribution-wise reward principle allows to cover all risk situations. 
Figure 4: First-order stochastic dominance for betting

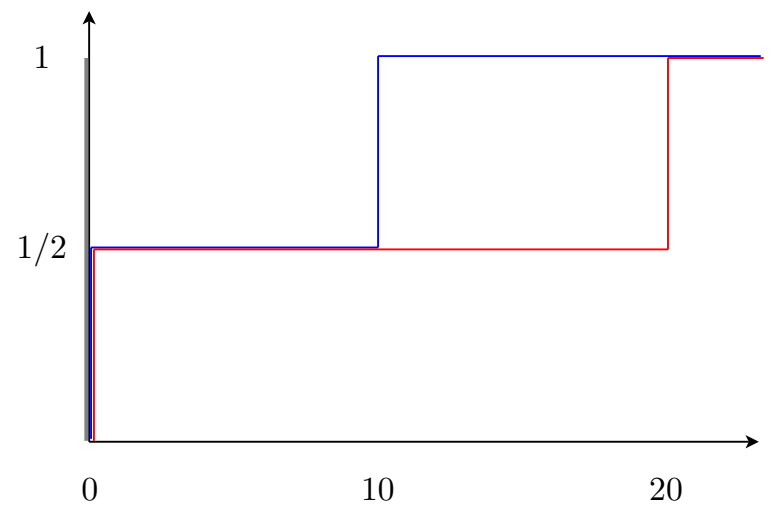

We will proceed by means of an example. Let us consider the case of betting.

Consider flipping a coin with three possible stakes 0,5 and 10 . We consider a fair bet and then if lucky you gain 10 if you bet 5 and 20 if you bet 10. The ranking of the efforts is non ambiguous, $0<5<10$. The CDF of the prospects are drawn in the Figure 5.2 and it appears that the CDF for the higher effort (betting 10) first-order stochastic dominates the CDF for the intermediate effort (betting 5) and the CDF for the null effort (the spike at 0). The CDFs which are drawn in Figure 4 are the CDFs corresponding to the gross outcome.

Obviously, it is well-known that in this example, the ranking of the distribution of net outcomes will be exactly the opposite in terms of second-order stochastic dominance (SSD), that is, the distribution of net prospects if not betting will SSD dominate the distribution of net prospects if betting 5 and so on.

We claim that, far from being a restrictive case, this example conveys a widespread intuition about risky situations. If we deliberately get rid of the undertaking cost, it must be that gross-income prospects corresponding to greater effort should first-order stochastic dominates gross-income prospects for lower effort. It is a necessary condition for investment in prevention to be rewarding. For this intuition to be valid, we must be prepared to be flexible enough to cope with a positive or negative view of undertaking risks. In the instance of binge drinking, we may endorse a negative view leading to consider that not drinking represents a greater effort than drinking. The effort could be measured by the number of glasses that you resist to drink. The more you abstain, the greater the effort.

To summarize, the two reward principles impose distinct restrictions on the effect of effort on individual outcomes. These restrictions differ in the way the correlation between effort and 
luck is taken into consideration when judging the effect of effort on outcomes. The point-wise reward principle only focuses on the direct effect of effort on outcome. On the contrary, the distribution-wise principle requires that a rise in effort improves the prospect for outcome, through two possible effects: the direct effect of effort on outcome and the indirect effect of effort on the distribution of luck.

Without further assumptions, the two principles are independent. However, if there is no correlation between the distribution of effort and luck, both principles coincide, as stated by the two following propositions.

Proposition 1 If the distribution of $l$ is independent of $e, P R$ implies $D R$.

Proof. By definition, $F_{Y \mid C, E}(y \mid c, e)=\int_{l \in \mathcal{L}(y, c, e)} f_{L \mid C, E}(l \mid c, e) d l$. Under the assumption of independence, the integrand in the equation for $F_{Y \mid C, E}(y \mid c, e)$ is independent of $e$. Since PR holds, if $e>e^{\prime}$ then $\mathrm{Y}(c, e, l) \geq \mathrm{Y}\left(c, e^{\prime}, l\right)$. This implies that $\mathcal{L}(y, c, e) \subset \mathcal{L}\left(y, c, e^{\prime}\right)$ for $e>e^{\prime}$ : with lower effort $e^{\prime}$ the range of admissible luck draws compatible with outcome lower than $y$ is larger. Hence the result.

The converse proposition is hardly more difficult to prove.

Proposition 2 If the distribution of $l$ is independent of $e$, if luck is uni-dimensional and if $\mathrm{Y}$ is monotonically increasing in $l, D R$ implies $P R$.

Proof. If $l$ is uni-dimensional and if $\mathrm{Y}$ is monotonically increasing in $l$ (which requires here that $\mathcal{L}$ be a metric space), the income rank in the distribution of $F_{Y \mid C, E}(\mid c, e)$ is identical to the luck rank in the distribution $F_{L \mid C, E}(\mid c, e)$. Furthermore, if the distribution of $l$ is independent of $e$, we have: $\forall e, f_{L \mid C, E}(l \mid c, e)=f_{L \mid C}(l \mid c)$. Hence, under the above assumptions, two individuals with similar circumstances and different effort who sit at the same rank of their income distributions, conditional on effort and circumstances have enjoyed similar luck $l$.

Define $F_{Y \mid C, E}^{-1}(q \mid c, e)$ the quantile function, conditional on $c$ and $e$. DR implies that for any $e>e^{\prime}$ and any $q \in[0,1], F_{Y \mid C, E}^{-1}(q \mid c, e) \geq F_{Y \mid C, E}^{-1}\left(q \mid c, e^{\prime}\right)$.

Noting that $F_{Y \mid C, E}^{-1}(q \mid c, e)=\mathrm{Y}(c, e, l)$ for $l=F_{L \mid C}^{-1}(q \mid c)$, we get: $\forall c, \forall e>e^{\prime}, \forall l \mathrm{Y}(c, e, l) \geq$ $\mathrm{Y}\left(c, e^{\prime}, l\right)$.

The condition that luck be uni-dimensional is not necessary, strictly speaking. We could investigate replacing this condition by some restrictions, on the outcome function or on the joint distribution of the components of luck, that would allow to generalize proposition 2 to a multidimensional luck environment. In a one-dimensional context, it is quite natural to think that 
the outcome function will be increasing in luck, because good luck means higher outcome. Increasingness is necessary in proposition 2 to identify luck on the basis of the outcome rank. However, in the case of proposition 1 increasingness is not needed: since the condition PR is satisfied for each value of $l$, the property survives by aggregation over values of luck, even if increasingness is violated (provided, of course that the distribution of luck is independent of effort).

The distribution-wise compensation principle LPT already propose the ex-ante version of the compensation principle. It rests on the view that differences in luck across individuals should not necessarily be preserved, contrary to differences in effort. They suggest that luck factors should be subject to a limited form of compensation: equality of opportunity does not require that the effect of luck be nullified (which may be impossible to implement without a perfect knowledge of the decision tree of all individuals in society) but that luck factors are even-handedly distributed, given circumstances and effort. Hence, the compensation principle should hold across all individuals who exert similar effort, regardless of their luck and for all possible circumstances. This view defines the distribution-wise conditional equality principle (DC):

Definition 4 The distribution-wise compensation principle $(D C)$ is satisfied iff: for any $\left(c, c^{\prime}\right) \in$ $\mathcal{C}^{n} \times \mathcal{C}^{n}$, for any $e \in \mathcal{E}^{p}$,

$$
F_{Y \mid C, E}(\mid c, e)=F_{Y \mid C, E}\left(\mid c^{\prime}, e\right)
$$

The difference in the two versions of the compensation principle stems from the possible influence of individual circumstances on luck. Given effort, the point-wise principle allows for differences in outcomes across individuals with distinct circumstances, but only to the extent that these differences are mediated by differences in luck. On the contrary, the distribution-wise principle rules out all differences in outcome that can be linked, directly or indirectly, to differential circumstances. It is also important to note that in the two principles, outcome comparisons are always restricted to individuals with similar effort. Lastly, without further assumptions, the two principles are independent. Of course, absent the correlation between circumstances and luck, the two compensation principles coincide.

Proposition 3 If the distribution of $l$ is independent of $c$ PC implies DC.

Proof. Define $\mathcal{L}(y, c, e)=\left\{l \in \mathcal{L}^{r} \mid \mathrm{Y}(c, e, l) \leq y\right\}$.

By definition, $F_{Y \mid C, E}(y \mid c, e)=\int_{l \in \mathcal{L}(y, c, e} f_{L \mid C, E}(l \mid c, e) d l$. The independence assumption 
implies that the integrand in the previous expression is independent of $c: \forall c, f_{L \mid C, E}(l \mid c, e)=$ $f_{L \mid E}(l \mid e)$. Assuming PR is satisfied further implies that the domain of integration is also independent of $c$. Hence, the result.

The converse proposition is not always true if luck is multi-dimensional.

Proposition 4 If the distribution of $l$ is independent of $c$, if luck is uni-dimensional and if $\mathrm{Y}$ is monotonically increasing in l, DC implies $P C$.

Proof. proof by contradiction. Assume that PC is not satisfied in the neighborhood of some $l^{\star}$ for some effort $e$. More precisely, assume that $\mathrm{Y}(c, e, l)=\mathrm{Y}\left(c^{\prime}, e, l\right)$ for $l \leq l^{\star}$ and that $\mathrm{Y}(c, e, l) \geq \mathrm{Y}\left(c^{\prime}, e, l\right)$ for $l \in\left[l^{\star}, l^{\star}+u\right]$ with $u$ arbitrarily small and the inequality is strict for at least one $l$. We can show by integration that: $F_{Y \mid C, E}(y \mid c, e)=F_{Y \mid C, E}\left(y \mid c^{\prime}, e\right)$ for $y \leq \mathrm{Y}\left(c, e, l^{\star}\right)$ and $F_{Y \mid C, E}(y \mid c, e)<F_{Y \mid C, E}\left(y \mid c^{\prime}, e\right)$ for $y=\mathrm{Y}\left(c, e, l^{\star}+u\right)$.

This result (DC is a sufficient condition of PC) does not generalize to multi-dimensional cases as demonstrated by the following example: two dimensions of luck, $l_{1}$ and $l_{2}$; two circumstances $c$ and $c^{\prime} ; \mathrm{Y}\left(c, e, l_{1}, l_{2}\right)=e l_{1} ; \mathrm{Y}\left(c^{\prime}, e, l_{1}, l_{2}\right)=e l_{2}$. Obviously, $\mathrm{PC}$ is violated since it is not true that for any pair $\left(l_{1}, l_{2}\right) \mathrm{Y}\left(c, e, l_{1}, l_{2}\right)=\mathrm{Y}\left(c^{\prime}, e, l_{1}, l_{2}\right)$. However, assume that each of the two dimensions of luck, $l_{1}$ and $l_{2}$, are drawn independently from a similar distribution law. Then in this case $F_{Y \mid C, E}(y \mid c, e)=F_{Y \mid C, E}\left(y \mid c^{\prime}, e\right)$ and DC is satisfied.

The case where effort is defined in a relative way has interesting implications for checking the distribution-wise principle of compensation. In this case, effort is distributed identically across all types. It is possible to reformulate the compensation principle as a constraint on the distribution of outcome within types. Define $F(y \mid c)$ the cumulative distribution of outcome in the population conditional on circumstances $c$. We can establish the following proposition.

Proposition 5 If effort is independent of circumstances, the distribution-wise compensation principle requires : for any $\left(c, c^{\prime}\right) \in \mathcal{C}^{n} \times \mathcal{C}^{n}$, for all $y \in \mathbb{R}^{+}, F(y \mid c)=F\left(y \mid c^{\prime}\right)$

\section{Proof. See LPT}

Taken together, propositions 1 to 4 imply that if luck is unidimensional and is uncorrelated to both circumstances and effort, the ex-ante and ex-post views are identical: PC and PR is equivalent to DC and DR. This will be the case when luck is measured as suggested by Fleurbaey (2008) by the percentile in the conditional distribution $F_{Y \mid C, E}(y \mid c, e)$ where the individual ends up. With such a measure, luck is obviously uncorrelated with $c$ and $e$. This measure is obviously 
consistent with some views of luck. For instance, our previous discussion of how randomness in deliberate gambles should be accounted fits this framework. Note though that not all forms of luck can be assumed to satisfy this absence of correlation with circumstances and effort, as illustrated by our previous discussion of moral hazard and genetic luck. In general, whether distribution-wise or point-wise principles should be used to characterize equality of opportunity amounts to making choices on how the correlation between circumstances, effort and luck should be regarded from an ethical perspective. We now address this point in the following section.

\section{Discussion}

The previous sections have left us equipped with two main tools: the first one is a three-way partitioning of the determinants of outcomes into effort, circumstances and luck; the second one is a pair of principles concerning the effect of circumstances and effort. These principles have been formulated under two perspectives: ex-post and ex-ante. We know have to discuss two main issues. The first pertains to the choice of the ex-post or ex-ante perspective. The second one asks whether the effect of luck on the distribution should be constrained by an explicit principle.

\subsection{The correlation between luck, circumstances and effort}

The main divide between the ex-ante and ex-post perspectives lies in the way they account for the correlation between luck on the one hand, and circumstances and effort on the other hand. This can be understood from the way luck appears in the definition of the principles, under each perspective.

In the point-wise principles, lucks appears explicitly as a conditioning variable, alongside circumstances and effort. So we fully condition on the level of luck when formulating each principle. In fact, luck stands in an intermediate position between effort and circumstances and appears in both principles. In the compensation principle, since luck is taken as a conditioning variable, the possible correlation between luck and circumstances is counted as luck and is excluded from the scope of compensation. Symmetrically, luck appears as conditioning variable in the reward principle, together with circumstances. This implies that the requisite of reward applies for every level of luck. Henceforth, the possible correlation between effort and luck is not counted as a possible source of reward. 
Table 1: Sorting of luck types and EOp principles

\begin{tabular}{|l|c|c|}
\hline & $E \cap L$ counts as effort & $E \cap L$ counts as luck \\
\hline$C \cap L$ counts as circumstances & DC, DR Moral Hazard & DC, PR Accidental luck \\
\hline$C \cap L$ counts as luck & PC, DR (?) & PC, PR Genetic talent \\
\hline
\end{tabular}

On the contrary, in the distribution-wise principles, luck only enters the principles implicitly, through its correlation with effort or circumstances. The compensation principle states that the effect of circumstances on the distribution of outcome, conditional on effort alone, should be nullified. Note here that in this formulation, luck does not appear explicitly. However, this amounts to requesting that luck affects the distribution of outcome in a neutral way w.r.t circumstance. This implies that the statistical association between luck and circumstances now falls on the compensation side and is counted as circumstances. Symmetrically, in the formulation of the reward principle, only circumstances are held constant. Hence, the reward to higher effort may arise from a positive statistical association with luck, as previously discussed. Put differently, the part of luck that is correlated with effort is included in the reward of effort. The views endorsed regarding the statistical association between luck and circumstances and effort, in the definition of each principle can be taken independently. In fact, in the compensation principle, the correlation between luck and effort is irrelevant. Similarly, in the reward principle, the correlation between luck and circumstances is irrelevant. For instance, one may combine the distribution-wise reward principle and the point-wise compensation principle. The above discussion suggests four distinct ways of defining equality of opportunity, depending on judgements about how the correlation between luck on the one hand, and circumstances and effort on the other hand, should be treated. Can we find a type of luck that corresponds to each cell of the table? Almost. Let us consider the three types of luck occurring at different moments of the timeline of Figure 3 genetic luck, accidental luck and moral hazard.

The proposed assignment of the different types of luck to the ex-ante and ex-post perspectives is presented in Table 1 below.

We have already argued that for moral hazard (entrepreneurial luck providing an example) we should apply the distribution-wise reward principle and treat the correlation between luck and effort as a component of effort. Let us now turn to the correlation between luck and circumstances. In the case of entrepreneurial luck, it might also be the case that the return on entrepreneurial activities depend on circumstances, through, for instance, family connections. In this case, compensation for circumstances should probably be extended to the benefits or 
disadvantages of circumstances that occur through the fact that individuals draw luck from a more or less favorable distribution of returns. In other words, we might want to leave individuals bear the consequences of their risky choice but net of the effect of circumstances. In this case, we would consider that the correlation between luck and circumstances should be considered as a circumstance and apply the distribution-wise compensation principle.

Now consider the case of accidental luck where luck is determined before effort is chosen. This could for instance correspond to luck in the marriage market which might intervene before labor market effort is decided but after circumstances. It might be the case that better luck induces individuals to expand more effort. For instance, outcomes might depend positively on individual's luck on the marriage market and society might decide that this form of luck does not in itself call for compensation. Still, at the same time, the correlation of this accidental luck with circumstances should certainly be considered as a circumstance and calls for a compensation. Now consider two individuals with the same intrinsic disposition for effort but with different accidental luck. However, as a result of accidental luck, one will end up expanding more effort. The reward for additional effort will be guaranteed anyway by some reward principle. Now the issue is to know whether he is more deserving because more lucky. The answer is no because luck happens before effort. Hence in this case, we should condition on luck in the formulation of the reward principle and apply the point-wise reward principle, i.e. treat the correlation between luck and effort as luck.

Yet in other cases, on the contrary, some might want to give priority to luck over circumstances. For instance, some people might agree that individuals should be allowed to enjoy the benefits of their innate ability, say a particular gift for music, or genetic endowment, such as beauty, irrespective of the family background they were born to. Suppose that all alleles linked to the physical appearance are observable. An earnings premium is attached to physical appearance: According to Hamermesh \& Biddle (1994) this premium is about $6 \%$ of earnings for good looking for men and $12 \%$ for women. Do we want to fully neutralize the likely correlation between social background and beauty or do we consider that it is a "natural" inequality? In this case, the possible correlation between genetic luck and circumstances should be treated as luck and one should apply the point-wise compensation principle. What about the correlation between beauty and effort? For the very reason that innate talent likely precedes effort, it will be difficult to assimilate the correlation between effort and genetic luck as effort. Hence, the application of the point-wise reward principle seems enough. 
As a matter of rule, we suggest that if luck precedes effort, the correlation between effort and luck will be counted for luck. On the opposite, if luck is posterior to effort, the correlation between effort and luck will be counted for effort. As regards the correlation between luck and circumstances, if luck precedes circumstances, then it appears as luck whereas if it is posterior to circumstances it will be considered as circumstances. A question mark appears in the above table in the bottom left cell. Indeed, this reasoning prevents to find a luck factor that is considered as a circumstance (it should be anterior to circumstance) and as an effort (it should be posterior to effort), since circumstances precedes luck.

\subsection{Luck: Beyond EOp}

To some extent, the requirements of EOp in the presence of luck, that we submitted in this paper, may be viewed as a weakening of the standard EOp principles. In the dual world of most EOp theories, there exist two categories of determinants of outcome and the effect of each set of determinants is constrained by distributive principle: the reward principle for effort and the compensation principle for circumstances. In our proposal, the two principles remain but we now have three categories. In fact, the distributional effect of luck is not constrained a priori. On the contrary, luck captures those determinants that escape the scope of the reward and compensation principles. This remark calls for several comments.

One should first emphasize the fact that the compensation principle implies that the correlation between luck and circumstances will be nullified, which in the real world, is violated in many instances. In many spheres of the socio-economic life (e.g. in education, on the labor market, in politics) individual outcomes may be described as the result of tournaments whose outcome depends on a combination of luck, circumstances and effort. A policy that would implement the distribution-wise compensation principle would probably be viewed by many as a formidable accomplishment.

Now, assume that this has been achieved and let us focus now on people belonging to the same type and exerting the same effort. They may be more or less successful as a result of luck and it is true that our principles do not require redistributing from the luckier to the less lucky. Observe nevertheless that the principles do not preclude either a full redistribution and can accommodate additional redistributive views. We admit that it can be disturbing for many that a theory of social justice such as Equality of Opportunity remains mute with respect to the effect of luck, and all the more so that EOp is often referred to, among philosophers, as 
luck-egalitarianism (Anderson 1999). However, this criticism is not totally fair since we have argued in the course of the paper that our framework is sufficiently flexible to consider bad accidental or bad genetic luck as circumstances and then to advocate for full compensation of these factors.

It should be also added that in the intra-personal dimension, individuals can buy private insurance in the market to smooth the effect of luck on her streams of outcomes across the life-cycle (for instance in the case of accidental luck such as health shocks). Her welfare will be improved and we can appeal to standard public-economics argument to subsidize private insurance if there is a market failure, i.e. insufficient private insurance provided by the market (or too expensive). Pareto optimally can be called in without any need to resort to a social justice argument. Instead of subsidizing private insurance, we can even recommend to deliver compulsory public insurance, in particular in case of moral hazard (for instance unemployment) where the failure of private market is very likely.

Lastly, along the inter-personal dimension, we agree that our framework may be viewed as incomplete as far as inequality among individuals with similar type and effort are concerned. We further admit that one should resort to principles of social justice external to the theory of equality of opportunity to ground a value judgement on the degree of inequality arising from luck. As food for thought, one may appeal to an externality argument. If income disparity among equals (persons with similar effort) becomes huge, they may ultimately not have the same real power in a democratic society and this will undermine democracy, with the risk that this political regime degenerates in a plutocracy. This is the essence of the externality argument that should be elaborated in more detail to complete EOp regarding the impact of luck. This argument may connect to that of Anderson (1999) and Scheffler (2003) who propose an alternative to luck egalitarianism they call 'democratic equality' which involves treating all persons with dignity and respect.

\section{Conclusion}

In this paper, we have argued that the strict dichotomy between effort and circumstances provides a restrictive framework for characterizing equality of opportunity, defined as a combination of a compensation principle and a reward principle. We submit that a comprehensive definition of equality of opportunity needs to take into account a third generic category, which we call luck 
and that should be distinguished from effort and circumstances. Luck gathers the factors that (i) do not call for specific compensation and (ii) do not imply any specific reward requirement. The main reason for endorsing such a proposition is that effort might stochastically influence the realization of some individual outcomes, as in moral hazard. As such, these determinants cannot be treated as circumstances or effort strictly speaking. Individual are only "partly responsible" for them. They call for compensation but only to the extent that they are related to individual circumstances. They should be respected, to the extent that they are shaped by effort choice. Lastly, they should not be seen in and of themselves, as a source of merit in the formulation of reward principles.

One may contend that the principles of equality of opportunity presented in this paper only provide an incomplete characterization of social justice. However, our proposal should rather be seen as an extension of the pragmatic approach to equality of opportunity defended by John Roemer in his (1993) paper. In this article, Roemer offers to put an end to the seemingly endless debate on the scope of individual responsibility by assuming that society has agreed on the set of individual circumstances. In his proposal, all other determinants are gathered under the term effort and a principle of compensation is constructed to define equality of opportunity. In the present paper, we substantiate the notion of effort and complement the Roemerian framework by, first, offering a simple reward principle and, second, by challenging the conception that all factors not included in circumstances should be considered as effort, in the application of this reward principle. Defining the set of factors that should be nullified falls short of identifying those factors that, according to society, should be rewarded. Here, we leave to society the burden of defining what these factors are and which factors should fall in the grey zone of luck, between circumstances and effort.

Of course, these definitions are provided under the assumption that the determinants of individual outcomes are fully observable. In practice, this will rarely be the case. Hence it would be necessary to discuss how the various notions of equality of opportunity examined in this paper could be identified empirically under observational constraints. We leave this discussion to future developments.

\section{References}

Anderson, E. (1999). What is the point of equality?, Ethics 109(2): 287-337.

Arneson, R. (1989). Equality and equal opportunity of welfare, Philosophical Studies 56: 77-93. 
Arneson, R. (2004). Luck egalitarianism interpreted and defended, Philosophical Topics 32(2): $1-20$.

Brown, J. E., Chatterjee, N., Younger, J. \& Mackey, S. (2011). Towards a physiology-based measure of pain: Patterns of human brain activity distinguish painful from non-painful thermal stimulation, PLOS ONE 6(9): 1-8.

Cappelen, A. W. \& Tungodden, B. (2006). A Liberal Egalitarian Paradox, Economics and Philosophy 22(03): 393-408.

Chiu, H. (2010). Skewness preference, risk-taking and expected utility maximisation, Geneva Risk and Insurance Review 2: 108-129.

Cohen, G. A. (1989). On the currency of egalitarian justice, Ethics 99: 906-944.

Cunha, F. \& Heckman, J. (2007). The technology of skill formation, American Economic Review 97(2): 31-47.

Dworkin, R. (1981a). What is equality. part 1: Equality of welfare, Philosophy and Public Affairs 10: 185-246.

Dworkin, R. (1981b). What is equality. part 2: Equality of ressources, Philosophy and Public Affairs 10: 283-345.

Dworkin, R. (2000). Sovereign Virtue: The Theory and Practice of Equality, Harvard University Press.

Fleurbaey, M. (2008). Fairness, Responsibility and Welfare, Oxford University Press, Oxford.

Fleurbaey, M. (2010). Assessing risky social situations, Journal of Political Economy 118(4): 649-680.

Fleurbaey, M. \& Schokkaert, E. (2009). Unfair inequalities in health and health care, Journal of Health Economics 28(1): 73-90.

Frank, R. (2016). Success and Luck: Good Fortune and the Myth of Meritocracy, Princeton University Press.

Garcia-Gomez, P., Schokkaert, E., Van Ourti, T. \& Bago d'Uva, T. (n.d.). Inequity in the face of death, Health Economics 24(10): 1348-1367.

Golec, J. \& Tamarkin, M. (1998). Gamblers favor skewness, not risk, at the horse track, Journal of Political Economy 106: 205-225.

Hamermesh, D. S. \& Biddle, J. E. (1994). Beauty and the labor market, The American Economic Review 84(5): 1174-1194.

Hurley, S. (2003). Justice, Luck and Knowledge, Oxford University Press, Oxford.

Kahneman, D., Wakker, P. P. \& Sarin, R. (1997). Back to Bentham? explorations of experienced utility, The Quarterly Journal of Economics 112(2): 375.

Le Grand, J. (1991). Equity and Choice, Harper Collins, London. 
Lefranc, A., Pistolesi, N. \& Trannoy, A. (2009). Equality of opportunity and luck: Definitions and testable conditions, with an application to income in france (1979-2000), Journal of Public Economics 93(11-12): 1189-1207.

Lefranc, A. \& Trannoy, A. (2017). The correlation between effort and circumstances in an uncertain world, mimeo .

Ramos, X. \& Van de gaer, D. (2016). Approaches to inequality of opportunity: Principles, measures, and evidence, Journal of Economic Surveys 30(5): 855-883.

Rawls, J. (1971). A Theory of Justice, Harvard University Press, Cambridge, MA.

Roemer, J. (1993). A pragmatic theory of responsibility for the egalitarian planner, Philosophy and Public Affairs 22(2): 146-166.

Roemer, J. (1998). Equality of Opportunity, Harvard University Press, Cambridge.

Savage, L. (1954). The foundations of statistics, Wiley, New York.

Scheffler, S. (2003). What is egalitrianism?, Philosophy \& Public Affairs 31(1): 5-39.

Vallentyne, P. (1997). Self-ownership and equality: Brute luck, gifts, universal dominance, and leximin, Ethics 107(2): 321-343.

Wager, T. D., Atlas, L. Y., Lindquist, M. A., Roy, M., Woo, C.-W. \& Kross, E. (2013). An fmri-based neurologic signature of physical pain, New England Journal of Medicine 368(15): 1388-1397. PMID: 23574118. 